Matjaž Kotar, Makoto Fujishima, Gideon N. Levy, Edvard Govekar, Initial transient phase and stability of annular laser beam direct wire deposition, CIRP Annals Manufacturing Technology,

Volume 68,

2019,

Pages 233-236,

ISSN 0007-8506.

C 2019. This manuscript version is made available under the CC-BY-NC-ND 4.0 license http://creativecommons.org/licenses/by-nc-nd/4.0/

Link to published article:

https://www.sciencedirect.com/science/article/pii/S0007850619301507?via\%3Dihub

DOI: https://doi.org/10.1016/j.cirp.2019.04.118 


\title{
Initial transient phase and stability of annular laser beam direct wire deposition
}

\author{
Matjaž Kotar a, Makoto Fujishima (3) ${ }^{\mathrm{b}}$, Gideon Levy (1) ${ }^{\mathrm{c}}$, Edvard Govekar (1) ${ }^{\mathrm{a}}$ \\ a University of Ljubljana, Faculty of Mechanical Engineering, Ljubljana, Slovenia \\ b DMG Mori, CO., LTD., 2-35-16 Meieki, Nakamura-ku, Nagoya City Aichu 450-0002, Japan \\ c TTA Technology, Switzerland
}

\begin{abstract}
In this paper the stability of the annular laser beam (ALB) direct wire-deposition process, which enables process symmetry and a well-defined ALB workpiece irradiation proportion (WIP) and related energy input onto the workpiece and the wire surface is considered. Various initial process phase strategies with respect to different initial wire-end positions and WIPs were analysed based on the process visualization and outcome, and the melt pool temperature. It was shown that in addition to a precise synchronization of the mutually time-dependent ALB power, wire and workpiece feeding velocity, the fastest and the most robust transition into a stable stationary process could be achieved with the initial position of the wire-end on the workpiece surface. Additionally, the WIP was shown to have a strong and nonlinear influence on the process stability.
\end{abstract}

\section{Introduction}

Laser direct metal deposition (L-DMD) is an additivemanufacturing (AM) process where a metal in the form of a powder or a wire is directed by a nozzle into a melt pool generated by a laser beam onto a metal surface [1]. The L-DMD process can be used in various AM applications, including 3D printing, coating, and the repair of 3D parts. The selection of the form of the material depends on the application and introduces several specific advantages and disadvantages. The main advantages of using a powder are the higher process stability and robustness, the wider material selection and the ability to mix several metal powders to create alloys and graded material parts [2]. On the other hand, the use of wire, rather than a powder, provides advantages, including the lower price of the wire material, the better material-use efficiency and the deposition rates, as well as there being no problems with handling safety and contamination of the environment. In the case of powder, this can be harmful to both humans and machines [1]. Additionally, due to the problem of oxidation, wire materials are easier to stock and are applicable for the deposition of reactive materials like $\mathrm{Al}$ and Ti alloys [2]. In order to perform laser direct wire deposition (L-DWD), in the simplest case a deposition head is used, by means of which the wire is fed laterally into the melt pool that is generated by a laser beam, directed perpendicular to the surface of the workpiece [1]. In this case the process performance and stability are influenced by the angle and direction of the lateral feeding, which can be either from the front, rear or side of the generated melt pool [1]. One of the main drawbacks of lateral feeding is the process asymmetry and the related directional dependence, which can be partly solved by a L-DWD head with a variable wire feeding direction [3]. Another approach to achieve L-DWD process symmetry, directional independence and better process stability is to feed the wire axially with the laser beam. This can be achieved either with several laser beams positioned around the axially fed wire [4] or by using an annular laser beam [5]. In spite of several advantages and improvements achieved with an axial wire feed, the related L-DWD process stability is still very sensitive to the process parameters [6], [7], especially in its initial transient phase.

In this paper the results of a stability analysis for an annular laser beam, axially fed, wire-deposition process are presented, focusing on the initial transient phase of the process. With this aim, in the following section the experimental setup, including the annular laser beam, direct wire-deposition (ALB-DWD) head is described first. This enables the symmetrical and simultaneous heating of the workpiece and the wire-end surface over its circumference using an annular laser beam. In order to characterize the proportion of the laser beam's energy input into the workpiece and the wire-end, introduced in [8], an experimental estimation of the workpiece irradiation proportion (WIP) is proposed. In the third section, different strategies for the initial transient phase of the ALB-DWD process, with respect to the wire-end initial position, are proposed and analysed, while in the fourth section the process stability is analysed.

\section{Annular laser beam wire-deposition setup and WIP}

The experimental setup for ALB-DWD, schematically shown in Fig. 1(a), consists of an ALB-DWD head, a wire-feeding unit, a workpiece translation stage, a process-monitoring system and a continuous, $2.5-\mathrm{kW}$, fiber laser source with a wavelength of $1080 \mathrm{~nm}$. Using a laser beam shaping unit in the ALB-DWD head $[8,9]$, a collimated laser beam is transformed into an ALB. By means of two reflective mirrors, the ALB is guided coaxially to the axis of the wire-guiding tube and focused on the workpiece surface using focusing optics. A metal wire is axially fed into the centre of the ALB using a wire-feeding unit consisting of a wire straightener, a wire feeder and a wire-guiding tube. A coaxial gas nozzle is used to deliver argon shielding gas around the wire-deposition zone on the surface of the workpiece. The workpiece standoff position (WSP) with respect to the ALB focus position, denoted by $h_{\mathrm{wp}}$, was determined using a laser distance sensor. In order to carry out the 


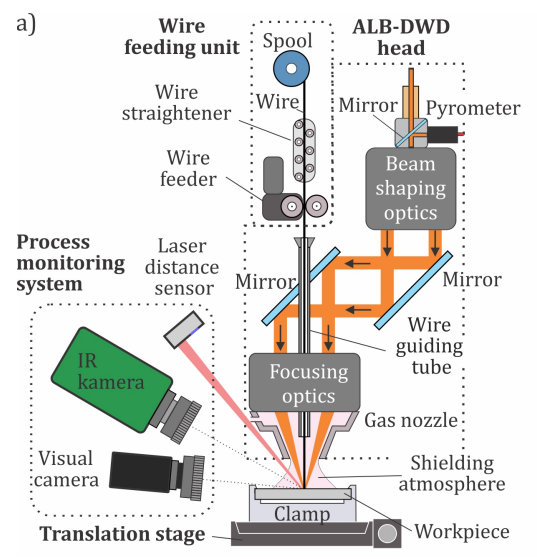

b)

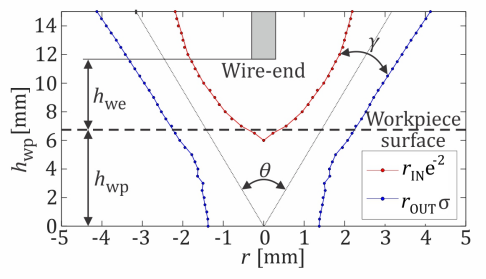

d)

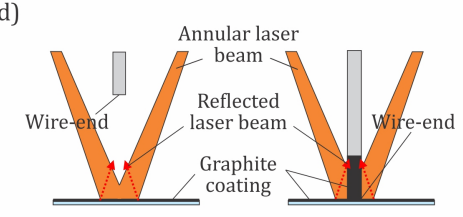

c)

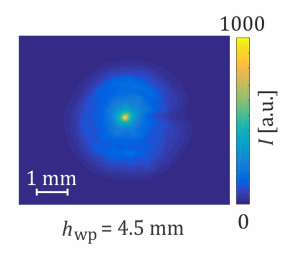

e)

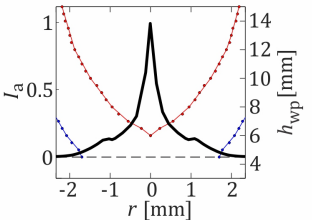

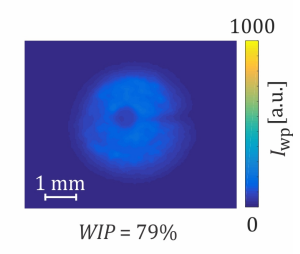

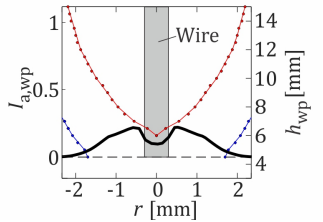

Figure 1.(a) ALB-DWD deposition set up, (b) ALB caustic, (c) Laser pulse thermal footprint $I$ and related $I_{\mathrm{a}}$ at $h_{\mathrm{wp}}=4.5 \mathrm{~mm}$. (d) Scheme of initial wire-end position for WIP measurement and initial process phase strategy. (e) Laser pulse thermal footprint $I_{\mathrm{wp}}$ and related $I_{\mathrm{a}, \mathrm{wp}}$ at $h_{\mathrm{wp}}=4.5 \mathrm{~mm}$ and $W I P=79 \%$.

ALB-DWD process visualization and melt pool temperature monitoring, a fast CMOS visual camera and a 2-colored in-axis pyrometer were used. Additionally, an IR camera was used for the ALB caustic and WIP characterization.

In Fig. 1(b) an example of an experimentally obtained ALB caustic above the focus position, which is applicable for the ALB-DWD process, with a convergence $\theta=15^{\circ}$ and a wedge angle $\gamma=1.7^{\circ}$ is presented. The red and blue dotted lines denote the inner $1 / \mathrm{e}^{2}$ and the outer $\mathrm{D} 4 \sigma$ laser beam intensity $I_{\mathrm{lb}}$ boundaries. The boundaries were estimated from a laser pulse thermal footprint intensity $I$ on a thin graphite layer, which was detected by the IR camera at various WSP values, without the presence of the wire in the ALB focus, as shown in Fig. 1(d)-left. An example of the thermal footprint intensity $I$ detected at WSP $h_{\mathrm{wp}}=4.5 \mathrm{~mm}$ and the related laser beam intensity profile $I_{a}(r)$ averaged over $360^{\circ}$ are shown in Fig. 1(c).

In the case of the wire's presence, as is shown schematically in Fig. 1(d)-right, an axisymmetric and simultaneous irradiation and heating of the wire and the workpiece surface can be achieved. The proportion of simultaneous workpiece and wire irradiation and related energy input, in general, depends on the ALB caustics, the WSP and the initial wire-end position, indicated by $h_{\text {we }}$ in Fig. 1(b). To characterize the simultaneous energy input into the workpiece and the wire, the process parameter WIP introduced in [8] is defined by:

$$
W I P=\frac{P_{w p}}{P}=\frac{\int_{0}^{r} b_{I} I_{a, w p}(r) 2 \pi r d r}{\int_{0}^{r_{b}} I_{a}(r) 2 \pi r d r} \cdot 100 \%,
$$

where $P_{\mathrm{wp}}$ and $P$ denote the proportion of the laser beam's power applied to the workpiece surface with and without the presence of the wire in the ALB focus. Based on the defined WIP, the proportion of the laser beam's power $P$ used to heat the wire-end is given by $(1-W I P)$. Using the second term of Eq. (1), $P_{\text {wp }}$ and $P$ can be calculated by integrating the average intensity profile $I_{\mathrm{a}, \mathrm{wp}}(r)$ and $I_{\mathrm{a}}(r)$ of the measured laser pulse thermal footprint intensity $I_{\mathrm{wp}}$ and $I$ detected with and without the presence of the wire in the ALB focus or simply by summing up the pixel values of the related IR images of the thermal footprint intensities $I_{\mathrm{wp}}$ and $I$.

An example of the laser beam's thermal footprint intensity $I_{\mathrm{wp}}$, detected at WSP $h_{\mathrm{wp}}=4.5 \mathrm{~mm}$ with the presence of a graphite coated wire-end at $h_{\mathrm{we}}=0.0 \mathrm{~mm}$, and the related laser beam intensity profile $I_{a, w p}(r)$ averaged over $360^{\circ}$, are shown in Fig. 1(e). It is clear that due to the simultaneous laser beam irradiation of the workpiece and the wire-end, a lower intensity $I_{\mathrm{wp}}$ and a smaller energy input into the workpiece surface are achieved. In the presented case, this yields $W I P=79 \%$.

\section{Initial transient phase and process stability}

The L-DWD process is considered to be stable as long the initially established molten bond (Fig. 2(a)) between the melt pool and the fed wire-end is sustained along the whole deposition path. This can be achieved by the proper energy input in space and time, which requires a precise time synchronization of the time-dependent laser beam power, wire feeding and workpiece scanning velocity. Process instability due to an improper energy input can, in general, result either in a collision of the unmelted wire-end and the workpiece surface (Fig. 2(b)) or in the formation of a pendent droplet (Fig. 2(c)), both of which lead to the unsuccessful formation or breaking of an already-established molten bond. The latter is especially important during the initial transient phase of the L-DWD process, which is essential for the further stability of the process. In the following three different initial phase strategies, considering two possible initial wire-end positions, i.e., above the workpiece surface, $h_{\mathrm{we}}>0.0 \mathrm{~mm}$, and on the workpiece surface, $h_{\mathrm{we}}=0.0 \mathrm{~mm}$, were considered and compared. In the experiments a SS 316 wire of $0.6-\mathrm{mm}$ diameter and SS 304 workpieces of dimensions $(w \times l \times h)=(25 \times 60 \times 10) \mathrm{mm}$ were used.

In Fig. 3, examples of graphs for the preset laser beam power $P(t)$, the wire $v_{\mathrm{w}}(t)$ and workpiece $v_{\mathrm{wp}}(t)$ feeding velocities and the measured melt pool temperature $T_{\mathrm{mp}}(t)$ of the three proposed initial phase strategies performed at WSP $h_{\mathrm{wp}}=3.5 \mathrm{~mm}$ are shown. The first two examples belong to the two considered initial strategies, 1a and b, with $h_{\mathrm{we}}>0.0 \mathrm{~mm}$. An advantage of these two strategies is that the melt pool can be generated without laser beam shadowing and power attenuation by the wire. However, rather uncontrolled irradiation of the wire-end by the reflected laser beam from the workpiece surface and later by the direct laser beam can take place. At a too high or a too low initial laser beam power $P_{0}$ this can lead either to the uncontrolled formation of a pendant droplet from the wire-end or to a collision of the wire-end and the workpiece surface. To overcome this problem, the initial wire-end position should be above the position of the reflected laser beam focus, as shown in Fig. 1(d)-left. Furthermore, in order to form a melt pool and a molten bond, using the strategy $1 \mathrm{a}$, an initially lower laser beam power $P_{0}$ of longer duration $t_{\mathrm{mb}}$ should be used, as is shown in Fig. 3(a), or using the strategy 1b, initially a
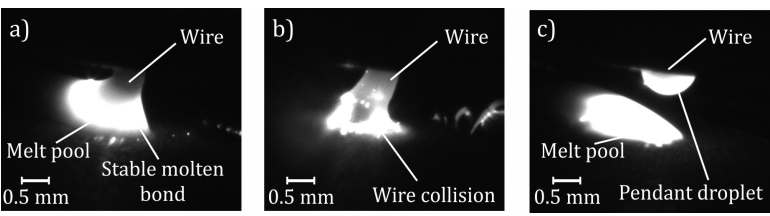

Figure 2. (a) Formed molten bond, (b) Wire collision, (c) Pendant droplet. 


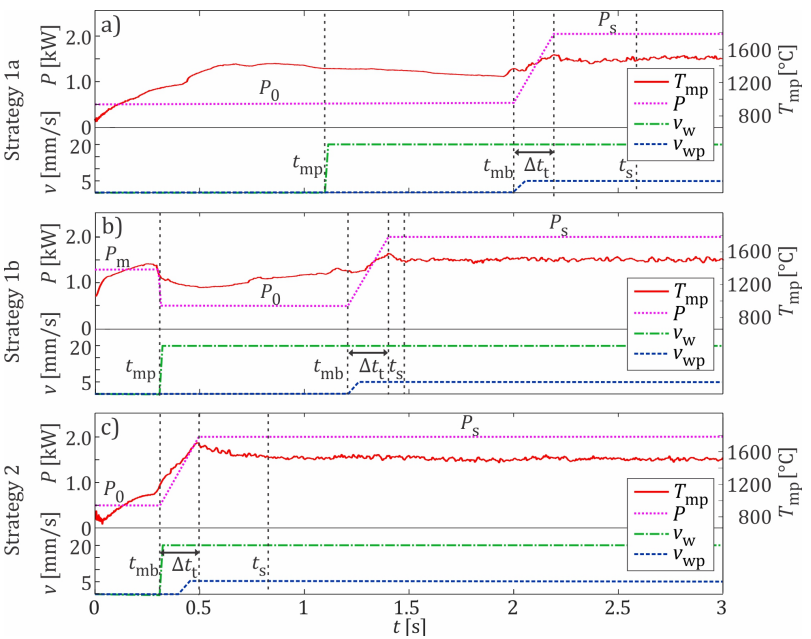

Figure 3. Initial strategies of ALB-DWD: (a) and (b) with initial wire-end position above the workpiece surface, and (c) on the workpiece surface.

laser beam of higher power $P_{\mathrm{m}}$ should be decreased to $P_{0}$ at time $t_{\mathrm{mp}}$, which corresponds to the moment of the melt pool formation, as shown in Fig. 3(b). At the time $t_{\mathrm{mp}}$ the feeding of the wire with a pre-set feeding velocity $v_{\mathrm{w}}$ and preheating of the wire-end take place up to the time $t_{\mathrm{mb}}$, at which a molten bond between the wireend and the workpiece surface is established. At the time $t_{\mathrm{mb}}$ of the molten bond formation, the feeding of the workpiece is also initiated with a pre-set feeding velocity $\nu_{\mathrm{wp}}$ and simultaneously the laser beam power $P$ is increased linearly to $P_{\mathrm{s}}$, within the time $\Delta t_{\mathrm{t}}$. With this, a high enough energy input is achieved to sustain the established molten bond and to ensure a smooth transition into the stationary phase of the process. The latter is clearly evident from the time course of the in-process-measured temperature $T_{\mathrm{mp}}$ of the melt pool, which at time $t_{\mathrm{s}}$ settles in both cases to around $T_{\mathrm{mp}, \mathrm{s}}=1530^{\circ} \mathrm{C}$. The values and relations of the process parameters $P_{0}, P_{\mathrm{m}}, P_{\mathrm{s}}, v_{\mathrm{w}}, v_{\mathrm{wp}}$ and the characteristic times $t_{\mathrm{mp}}, t_{\mathrm{mb}}$ and $\Delta t_{\mathrm{t}}$ are mutually dependent and complex due to the laser beam's interaction with the fed wire-end and the workpiece surface. In the considered examples, shown in Figs. 3(a) and 3(b), at pre-set wire and workpiece feeding velocities $v_{\mathrm{w}}=20 \mathrm{~mm} / \mathrm{s}$ and $v_{\mathrm{wp}}=5 \mathrm{~mm} / \mathrm{s}$ the values of $P_{0}, P_{\mathrm{m}}, P_{\mathrm{s}}$ and $t_{\mathrm{mp}}, t_{\mathrm{mb}} \Delta t_{\mathrm{t}}$ were obtained experimentally by an analysis of the records of the process visualisation and are given in Table 1 . In order to compare all three strategies, the same initial laser beam power $P_{0}=0.5 \mathrm{~kW}$ was used. We can see that with strategy $1 \mathrm{~b}$, due to the higher initial laser power $P_{\mathrm{m}}=1.3 \mathrm{~kW}$ the characteristic times $t_{\mathrm{mp}}, t_{\mathrm{mb}}$, and the related $t_{\mathrm{s}}$ of the transition into the stationary phase are shorter. To overcome the above drawbacks related to the initial position of the wire-end above the workpiece surface, the initial phase strategy 2 with the wire-end on the workpiece surface, as schematically shown in Fig. 1(d)-right, was proposed. In contrast to the previous two strategies, in this case the workpiece surface and the wire-end are heated simultaneously during the time interval $\left(0, t_{\mathrm{mb}}\right)$ with a laser beam of power $P_{0}$, and $W I P=92 \%$, causing the simultaneous formation of a melt pool and a molten bond. As shown in Fig. 3(c), after the moment $t_{\mathrm{mb}}$ of the establishment of the initial molten bond, the laser beam power $P_{0}$ is linearly increased to $P_{\mathrm{s}}$ and the feeding of the wire is started, to prevent interruption of the molten bond due to pendant-droplet formation. Furthermore, in order to ensure sufficient energy for the formation of a well-formed molten bond, the workpiece feeding is initiated with a slight delay during

Table 1. Process parameters and characteristic times.

\begin{tabular}{|c|c|c|c|c|c|c|c|}
\hline & \multicolumn{7}{|c|}{ Parameter } \\
\hline Strategy & $P_{\mathrm{m}}, \mathrm{kW}$ & $P_{0}, \mathrm{~kW}$ & $P_{\mathrm{s}}, \mathrm{kW}$ & $t_{\mathrm{mp}}, \mathrm{s}$ & $t_{\mathrm{mb}, \mathrm{s}}$ & $\Delta t_{\mathrm{t}}, \mathrm{s}$ & $t_{\mathrm{s}}, \mathrm{s}$ \\
\hline 1a & $/$ & 0.5 & 2.0 & 1.1 & 2.0 & 0.2 & 2.58 \\
\hline 1b & 1.3 & 0.5 & 2.0 & 0.3 & 1.2 & 0.2 & 1.47 \\
\hline 2 & $/$ & 0.5 & 2.0 & $/$ & 0.3 & 0.2 & 0.80 \\
\hline
\end{tabular}

the linear increase of the laser beam power, which ensures a smooth transition into the stationary phase of the process. From the time course of the melt pool temperature $T_{\mathrm{mp}}$, a significantly faster transition into the stationary process phase can be observed at time $t_{\mathrm{s}}=0.80 \mathrm{~s}$, whereas the melt pool temperature settles at $T_{\mathrm{mp}, \mathrm{s}}=1530^{\circ} \mathrm{C}$.

\section{Process-stability windows}

In the following, the results of the stability analysis of the ALBDWD process using strategy 2, i.e., with the initial position of the wire-end on the workpiece surface, are presented. For this purpose, an extensive set of experiments for a single-layer deposition of SS 316 wire of $0.6-\mathrm{mm}$ diameter onto a SS 304 workpiece was performed at different wire and workpiece feeding velocities, and varying the $W I P$ values in the range 40 to $100 \%$. In contrast to strategies $1 \mathrm{a}$ and $1 \mathrm{~b}$, in this case the value of the laser beam power $P_{0}$ depends on the WIP and the time $t_{\mathrm{mb}}$ of the molten bond formation only. In Fig. 4(a) the stability diagram of the laser beam power $P_{0}$ in the plane $P_{0}-W I P$ is shown for $t_{\mathrm{mb}}=0.3 \mathrm{~s}$. It is clear that with increasing WIP due to the lower energy input into the wire-end, the related laser beam power $P_{0}$ required to initiate the molten bond increases quasi-linearly. In the experiments, the maximum values $P_{0}$ were selected to initiate the molten bond. The selection of $P_{0}$ above the maximum value, at $t_{\mathrm{mb}}=0.3 \mathrm{~s}$, results in the formation of a pendant droplet before the molten bond could form and the selection of $P_{0}$ below the minimum value would require a longer $t_{\mathrm{mb}}$ to prevent any collision between the wire-end and the workpiece.

After the successful initiation of the molten bond in accordance with strategy 2 the laser beam power is linearly increased to $P_{\mathrm{s}}$. The experiments showed that at $\Delta t_{\mathrm{t}}=0.2 \mathrm{~s}$, which can be kept constant, the laser beam power $P_{\mathrm{s}}$ in the stable, stationary phase in addition to the WIP depends on the feeding velocities $v_{\mathrm{w}}$ and $v_{w p}$. In Figs. 5(a) and (b) the stability diagrams of the ALB-DWD process in the plane $P_{\mathrm{s}}-W I P$ are shown for wire feeding velocities $v_{\mathrm{w}}=10$ and $20 \mathrm{~mm} / \mathrm{s}$, where the blue color denotes the stable regions of the process. Within the stable region the blue stars and the red circles denote a stable process for workpiece feeding velocities $v_{\mathrm{wp}}=5$ and $10 \mathrm{~mm} / \mathrm{s}$. From the stability diagram for the wire feeding velocity $v_{\mathrm{w}}=10 \mathrm{~mm} / \mathrm{s}$ it is clear that the lower stability boundary and the related minimum laser beam power $P_{\mathrm{s}, \min }(W I P)$ as well the upper stability boundary and the related maximum laser beam power $P_{\mathrm{s}, \max }(W I P)$, required for a stable process, nonlinearly increase with an increase of the WIP. In relation to this, the width of the laser beam power stability interval $\left(P_{\mathrm{s}, \min }, P_{\mathrm{s}, \max }\right)$ increases until $W I P=96 \%$, after which the applied $P_{\mathrm{s}, \max }$ reaches a maximum output power of $2.5 \mathrm{~kW}$ for the laser source. In addition to the stable region, two qualitatively different L-DWD processinstability regions can be observed. At lower WIPs and laser beam powers $P_{\mathrm{s}}>P_{\mathrm{s}, \max }$, the process becomes unstable due to an excessively high energy input into the wire-end, which results in molten bond breaking and pendant-droplet formation. At higher $W I P$ values and a lower laser beam power $P_{\mathrm{s}}<P_{\mathrm{s} \text {, min }}$ the instability
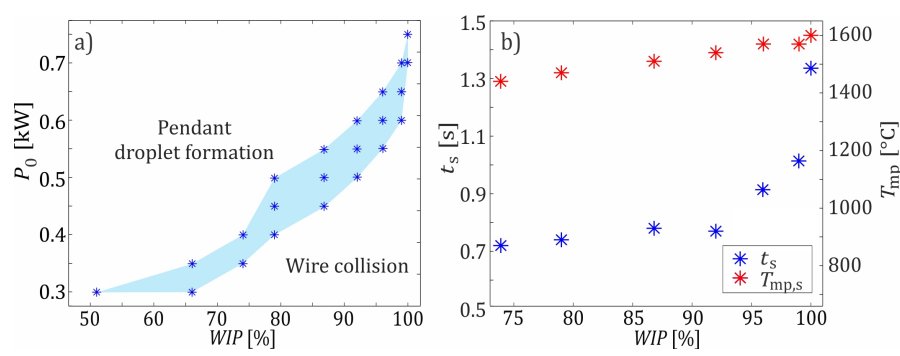

Figure 4. (a) Initial laser beam power $P_{0}$ stability interval vs. WIP at $t_{\mathrm{mb}}=0.3 \mathrm{~s}$. (b) Settling time $t_{\mathrm{s}}$ and the related melt pool temperature $T_{\mathrm{mp}, \mathrm{s}}$. 

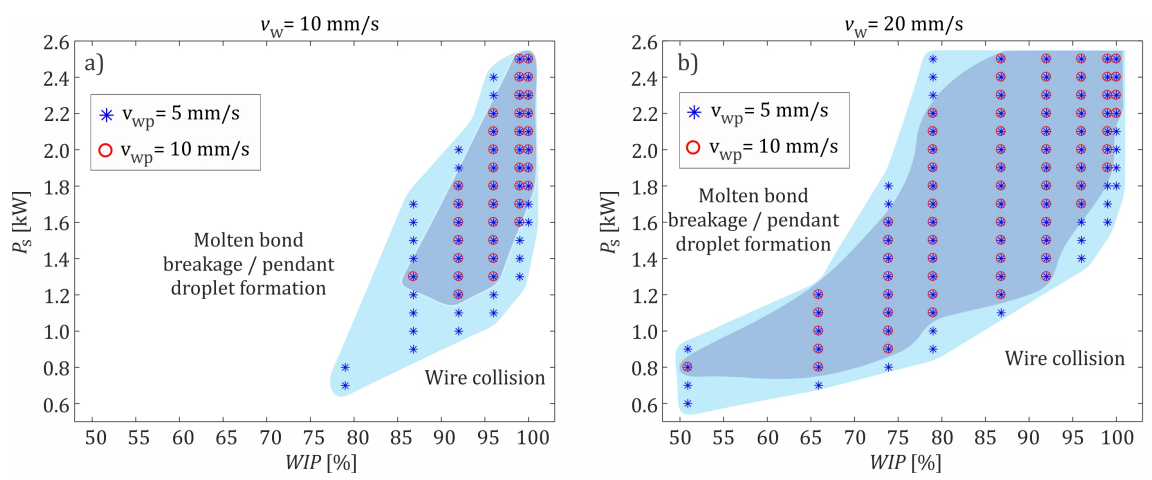
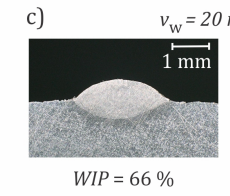

d)

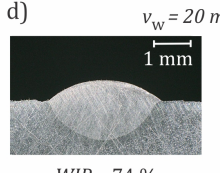

$W I P=74 \%$

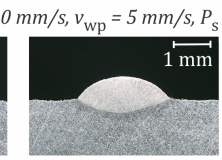

$W I P=74 \%$

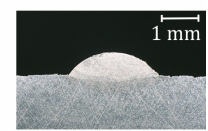

$W I P=87 \%$

$\mathrm{mm} / \mathrm{s}, v_{\mathrm{wp}}=5 \mathrm{~mm} / \mathrm{s}, P_{\mathrm{s}}=1.8 \mathrm{~kW}$

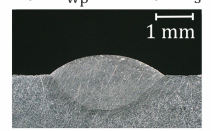

$W I P=92 \%$

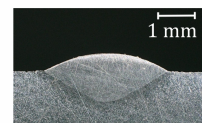

$W I P=100 \%$

Figure 5. Stability diagram at wire feeding velocity: (a) $v_{\mathrm{w}}=10 \mathrm{~mm} / \mathrm{s}$. (b) $\nu_{\mathrm{w}}=20 \mathrm{~mm} / \mathrm{s}$. Influence of the WIP on the deposited layer cross-section geometry at $P_{\mathrm{s}}=1.1 \mathrm{~kW}(\mathrm{c})$ and $P_{\mathrm{s}}=1.8 \mathrm{~kW}(\mathrm{~d})$.

due to a too low energy input into the wire-end takes place, which results in a collision between the wire and the workpiece. Furthermore, it can be seen that with an increase of the workpiece feeding velocity to $v_{\mathrm{wp}}=10 \mathrm{~mm} / \mathrm{s}$, the stability region denoted by the red circles shrinks. The related minimally required laser beam power $P_{\mathrm{s} \text {, min }}$ increases, whereas the maximum laser beam power $P_{\mathrm{s}, \max }$ and the related width of the stability interval $\left(P_{\mathrm{s}, \min }, P_{\mathrm{s}, \max }\right)$ decrease. As presented in Fig. 5(b), at a higher wire feeding velocity $v_{\mathrm{w}}=20 \mathrm{~mm} / \mathrm{s}$ the qualitative properties of the stability diagram are preserved. However, quantitatively, process stability can be achieved even at a lower WIP. Furthermore, the minimum $P_{\mathrm{s}, \min }$ and the maximum $P_{\mathrm{s}, \max }$ laser beam power as well as the width of the laser beam power process stability interval $\left(P_{\mathrm{s}, \mathrm{min}}\right.$, $\left.P_{s, \max }\right)$ are increased.

In all the observed cases, the nonlinear increase of $P_{\mathrm{s}, \min }(W I P)$ can be related to the fact that, with an increase of the $W I P$, a higher portion of the laser beam energy is introduced into the workpiece and therefore a higher laser beam power is required to achieve the melting of the wire-end, which is needed to establish a molten bond. Furthermore, the observed shrinkage of the stability region and the related interval $\left(P_{\mathrm{s}, \min }, P_{\mathrm{s}, \max }\right)$ with an increase in the workpiece feeding velocity $v_{\mathrm{wp}}$ can be explained by the fact that, with an increase of $v_{\mathrm{wp}}$, a larger laser beam power $P_{\mathrm{s} \text {,min }}$ is required in order to form the melt pool. On the other hand, the observed decrease in $P_{\mathrm{s} \text {, max }}$ could, presumably, be related to the additional heating of the wire by the laser beam, reflected from the generated melt pool, which results in process instability due to pendantdroplet formation at a lower $P_{\mathrm{s}}$. In Fig. 4(b) the settling time $t_{\mathrm{s}}$ and the related temperature $T_{\mathrm{mp}, \mathrm{s}} \mathrm{vs}$. WIP are shown at $P_{\mathrm{s}}=1.8 \mathrm{~kW}$ and at wire and workpiece feeding velocities $v_{\mathrm{w}}=20 \mathrm{~mm} / \mathrm{s}$ and $v_{\mathrm{wp}}=5 \mathrm{~mm} / \mathrm{s}$. It can be seen that with increasing WIP, the settling time $t_{\mathrm{s}}$ nonlinearly increases from 0.72 to $1.33 \mathrm{~s}$, and the related stationary melt pool temperature $T_{\mathrm{mp}, \mathrm{s}}$ linearly increases in the interval from 1490 to $1590^{\circ} \mathrm{C}$.

With the aim of showing the influence of the WIP on the deposited layer's geometrical properties and dilution, in Fig. 5(c) selected examples of the layer cross-section at wire and workpiece feeding velocities $v_{\mathrm{w}}=20 \mathrm{~mm} / \mathrm{s}$ and $v_{\mathrm{wp}}=5 \mathrm{~mm} / \mathrm{s}$, and laser beam powers $P_{\mathrm{s}}=1.1$ and $1.8 \mathrm{~kW}$ using WIP between 66 and $100 \%$ are shown. It can be seen qualitatively that the WIP influences the dilution of the deposited layer, which decreases with increasing WIP. Additionally, a comparison of the deposited layer at $W I P=74 \%$ and $P_{\mathrm{s}}=1.1$ and $1.8 \mathrm{~kW}$ shows the influence of the laser beam power $P_{\mathrm{s}}[10]$.

\section{Conclusions and discussion}

In the paper the stability of the annular laser beam direct wiredeposition (ALB-DWD) process with a focus on the initial transient phase of the process has been considered. Three different initial phase strategies with respect to the initial wire-end position, being either above or on the workpiece surface, were investigated. The experiments involving single-layer deposition of a SS 316 wire of 0.6 -mm diameter onto a SS 304 workpiece were characterized by the melt pool temperature and process visualization. The results showed that, in general, regardless to the initial strategy of the ALB-DWD process, the reason for the process instability is an improper energy input into the workpiece and the wire, which can result either in the collision of the wire-end and the workpiece, or in the breaking of the established molten bond and the formation of a pendant droplet at the wire-end. Using the initial strategy with the initial wire-end position on the workpiece surface makes it possible to have simultaneous heating of the workpiece and wire with a preset proportion defined by the workpiece-wire irradiation proportion (WIP). With this strategy the fastest and the most robust transition into a stable stationary phase of the process can be achieved. Furthermore, the results of the process stability and deposited layer cross-section analyses have shown that in addition to the standard process parameters the WIP significantly, and in a nonlinear manner, influences the process stability and its robustness, as well as the geometrical properties, i.e., mainly the dilution of the deposited layer.

\section{Acknowledgments}

The authors acknowledge the financial support of the Slovenian Research Agency (research core funding No. P2 0241) and DMG Mori Co., Ltd.

\section{References}

[1] Mok, S.H., Bi, G., Folkes, J., Pashby, I., 2008. Deposition of Ti-6Al-4V using a high power diode laser and wire, Part I: Investigation on the process characteristics. Surf. Coatings Technol. 202, 3933-3939.

[2] Bourell, D., Pierre, J., Leu, M., Levy, G., Rosen, D., Beese, A.M., Clare, A., 2017. Materials for additive manufacturing. CIRP Ann. - Manuf. Technol. 66, 659-681.

[3] Wu, Q., Lu, J., Liu, C., Shi, X., Ma, Q., Tang, S., Fan, H., Ma, S., 2017. Obtaining uniform deposition with variable wire feeding direction during wire-feed additive manufacturing. Mater. Manuf. Process. 32, 1881-1886.

[4] Pajukoski, H., Näkki, J., Thieme, S., Tuominen, J., Nowotny, S., Vuoristo, P., 2016. High performance corrosion resistant coatings by novel coaxial cold- and hot-wire laser cladding methods. J. Laser Appl. 28, 012011.

[5] M. J. Wu, S. H. Shi, G. Y. Fu, Y.L., 2013. Process Parameters Control of Deposition of Hollow Cylindrical Part with Internal Wire Feeding through a Hollow Laser Beam. Appl. Mech. Mater. 419, 305-309.

[6] Hagqvist, P., Heralić, A., Christiansson, A.K., Lennartson, B., 2015. Resistance based iterative learning control of additive manufacturing with wire. Mechatronics 31, 116-123.

[7] Heralić, A., Christiansson, A.K., Ottosson, M., Lennartson, B., 2010. Increased stability in laser metal wire deposition through feedback from optical measurements. Opt. Lasers Eng. 48, 478-485.

[8] Kotar, M., Govekar, E., 2018. The influence of the workpiece illumination proportion in annular laser beam wire deposition process. Procedia CIRP 74, 228-232.

[9] Govekar, E., Jeromen, A., Kuznetsov, A., Levy, G., Fujishima, M., 2018. Study of an annular laser beam based axially-fed powder cladding process. CIRP Ann. Manuf. Technol. 67, 241-244.

[10] Abioye, T.E., Folkes, J., Clare, A.T., 2013. A parametric study of Inconel 625 wire laser deposition. J. Mater. Process. Technol. 213, 2145-2151. 